UCLA/05/TEP/4 SLAC-PUB-11003 Saclay/SPhT-T05/015 hep-th/yymmnnn

\title{
On-Shell Recurrence Relations for One-Loop QCD Amplitudes*
}

\author{
Zvi Bern \\ Department of Physics and Astronomy, UCLA \\ Los Angeles, CA 90095-1547, USA \\ Lance J. Dixon \\ Stanford Linear Accelerator Center \\ Stanford University \\ Stanford, CA 94309, USA \\ David A. Kosower \\ Service de Physique Théorique $e^{\dagger}, C E A-S a c l a y$ \\ F-91191 Gif-sur-Yvette cedex, France
}

(Dated: January 28, 2005)

\begin{abstract}
We present examples of on-shell recurrence relations for determining rational functions appearing in one-loop QCD amplitudes. In particular, we give relations for one-loop QCD amplitudes with all legs of positive helicity, or with one leg of negative helicity and the rest of positive helicity. Our recurrence relations are similar to the tree-level ones described by Britto, Cachazo, Feng and Witten. A number of new features arise for loop amplitudes in non-supersymmetric theories like QCD, including boundary terms and double poles. We show how to eliminate the boundary terms, which would interfere with obtaining useful relations. Using the relations we give compact explicit expressions for the $n$-gluon amplitudes with one negative-helicity gluon, up through $n=7$.
\end{abstract}

PACS numbers: 11.15.Bt, 11.25.Db, 11.25.Tq, 11.55.Bq, 12.38.Bx

\footnotetext{
* Research supported in part by the US Department of Energy under contracts DE-FG03-91ER40662 and DE-AC02-76SF00515

$\dagger$ Laboratory of the Direction des Sciences de la Matière of the Commissariat à l'Energie Atomique of France.
} 


\section{INTRODUCTION}

Seeking out new physics beyond the standard $S U(3) \times S U(2) \times U(1)$ model of particle interactions is the goal of the experimental program at the forthcoming Large Hadron Collider (LHC) at CERN. The discovery, and study, of such new physics will depend on our ability to calculate a wide variety of processes in the gauge theories making up the Standard Model. While computations of tree-level scattering amplitudes are a first step, the size and scale-variation of the strong coupling constant imply that a basic quantitative understanding must also include the one-loop amplitudes which enter into next-to-leading order corrections to cross sections. Such corrections are required to build a theoretical base for a program of precision measurements at hadron colliders [1]. Precision measurements at the SLAC Linear Collider (SLC) and CERN's Large Electron Positron (LEP) collider have proven the power of such a program in advancing our understanding of short-distance physics.

Recently, there has been tremendous progress in understanding and computing gauge theory scattering amplitudes, stimulated by Witten's proposal [2] of a weak-weak coupling duality between $\mathcal{N}=4$ supersymmetric gauge theory and the topological open-string $B$ model, generalizing Nair's earlier description [3] of the simplest gauge-theory amplitudes.

While there are still open questions about the precise computation of tree-level amplitudes in the twistor string theory [4], and open questions about the structure of the theory at loop level [5], the proposal has already led to several important insights into the structure of gauge-theory amplitudes. In particular, it allowed Cachazo, Svrček and Witten (CSW) [6] to formulate a new set of vertices and rules for computing tree-level amplitudes in either supersymmetric or non-supersymmetric gauge theories. The vertices are off-shell continuations of the maximally helicity-violating (MHV) amplitudes, which were written down long ago by Parke and Taylor [7] — in amplitude form by Mangano, Parke and Xu [8] — and proven using recurrence relations by Berends and Giele [9]. (For a discussion of the connections between different tree-level pictures, see refs. [10,11].) A number of papers have applied these techniques to explicit calculations [12-14]. Recently, several authors have taken the first steps in applying such ideas to mixed QCD-electroweak amplitudes as well [15]. At loop level, earlier apparent complications [16] in the twistor-space structure of amplitudes were clarified through an explicit linking of CSW vertices to the unitarity-based method [17], and via the elucidation [18] and computation of the holomorphic anomaly [19, 20]. 
Motivated by early puzzles about the twistor-space structure of one-loop amplitudes, two groups pursued calculations of the next-to-MHV (NMHV) class of $n$-gluon amplitudes in the $\mathcal{N}=4$ super-Yang-Mills theory [21-25], primarily using the unitarity-based method [26-29]. A recent development, enhancing the power of the unitarity method, is the observation [23] that box integral coefficients can be obtained from generalized unitarity cuts [22, 29, 30] by solving the constraints that multiple intermediate states be on shell. (The notion of 'generalized unitarity', as applied to amplitudes for massive particles, can be traced back to ref. [31].) As a by-product of these calculations, ref. [22] revealed a simpler representation for the NMHV seven-gluon amplitude than had been known previously, from either a recurrencebased computation [32] or the CSW rules [6]. The NMHV computation for arbitrary values of $n$ [24], like that for $n=7$, made use of infrared consistency equations, which express the constraint that a one-loop amplitude must have the correct infrared poles [33] in the dimensional regulator $\epsilon$. Again as a by-product, the solutions to the equations revealed a new class of representations of tree amplitudes. A particularly nice one [34] gave the natural generalization of the simple representation to the eight-point amplitude, and suggested a new type of on-shell recurrence relation for tree amplitudes. Britto, Cachazo and Feng wrote down [35] the corresponding recurrence relation, which yields compact expressions for NMHV tree amplitudes [36]. The same authors and Witten (BCFW) gave a very simple and elegant proof [37] of the relation using special complex continuations of the external momenta.

The proof, which we review in section III, is actually quite general, and applies to any rational function of the external spinors satisfying certain scaling and factorization properties. The generality of the proof motivated us to find a loop version of the recurrence relation. In this paper, we shall apply it to the finite one-loop amplitudes in QCD, which are purely rational. We will also argue that it can be applied to compute the rational terms in other, cut-containing, QCD amplitudes. We shall see that the application to general loop amplitudes in a non-supersymmetric theory like QCD requires that we address new issues, such as double poles in the complex analytic continuation, which are related to properties of these amplitudes in the limit that two momenta become collinear.

Recurrence relations can provide a technique, complementary to the unitarity-based method, for the complete calculation of one-loop QCD amplitudes. The unitarity method applies most easily to terms in the amplitudes that have discontinuities. Computation of 
these terms requires only knowledge of tree amplitudes evaluated in four dimensions. The unitarity method can also be applied to computation of rational terms, because the latter effectively have cuts at higher order in $\epsilon$ [28, 29]; but doing so requires knowledge of tree amplitudes with two legs in $D=4-2 \epsilon$ dimensions, which are harder to compute. Another approach is to build up an ansatz for the rational terms, based on their collinear and multi-particle factorization properties. This approach has been successful for amplitudes with sufficiently simple structure [38, 39]; and in more complex cases, with assistance from other, numerical representations of the amplitudes [30]. However, it involves some guesswork and so it is not easy to systematize. An alternative, recurrence-based method, along the lines of the present paper, would represent a systematic method for constructing rational function terms in amplitudes directly from their poles. It thus holds forth the prospect of dramatically simplifying such calculations.

This paper is organized as follows. In section II we define the notation used in this paper. Section III reviews the BCFW proof. Then in section IV we construct recurrence relations for the all positive-helicity one-loop amplitudes. Section V presents such a relation for the one-loop amplitudes containing a single negative-helicity gluon, and also displays new compact forms for the cases of $n=6$ and $n=7$, obtained via the relation. Finally, in section VI we give our conclusions and outlook for the future.

\section{NOTATION}

We use the trace-based color decomposition [8, 40, 41] of amplitudes. For tree-level amplitudes with $n$ external gluons, this decomposition is,

$$
\mathcal{A}_{n}^{\text {tree }}\left(\left\{k_{i}, h_{i}, a_{i}\right\}\right)=\sum_{\sigma \in S_{n} / Z_{n}} \operatorname{Tr}\left(T^{a_{\sigma(1)}} \cdots T^{a_{\sigma(n)}}\right) A_{n}^{\text {tree }}\left(\sigma\left(1^{h_{1}}, \ldots, n^{h_{n}}\right)\right),
$$

where $S_{n} / Z_{n}$ is the group of non-cyclic permutations on $n$ symbols, and $j^{h_{j}}$ denotes the $j$-th momentum and helicity $h_{j}$. The $T^{a}$ are fundamental representation $\mathrm{SU}\left(N_{c}\right)$ color matrices normalized so that $\operatorname{Tr}\left(T^{a} T^{b}\right)=\delta^{a b}$. The color-ordered amplitude $A_{n}^{\text {tree }}$ is invariant under a cyclic permutation of its arguments.

We describe the amplitudes using the spinor helicity formalism. In this formalism amplitudes are expressed in terms of spinor inner-products,

$$
\langle j l\rangle=\left\langle j^{-} \mid l^{+}\right\rangle=\bar{u}_{-}\left(k_{j}\right) u_{+}\left(k_{l}\right), \quad[j l]=\left\langle j^{+} \mid l^{-}\right\rangle=\bar{u}_{+}\left(k_{j}\right) u_{-}\left(k_{l}\right),
$$


where $u_{ \pm}(k)$ is a massless Weyl spinor with momentum $k$ and plus or minus chirality [41, 42]. Our convention is that all legs are outgoing. The notation used here follows the standard QCD literature, with $[i j]=\operatorname{sign}\left(k_{i}^{0} k_{j}^{0}\right)\langle j i\rangle^{*}$ so that,

$$
\langle i j\rangle[j i]=2 k_{i} \cdot k_{j}=s_{i j}
$$

(Note that the square bracket $[i j]$ differs by an overall sign compared to the notation commonly used in twistor-space studies [2].)

We denote the sums of cyclicly-consecutive external momenta by

$$
K_{i \cdots j}^{\mu} \equiv k_{i}^{\mu}+k_{i+1}^{\mu}+\cdots+k_{j-1}^{\mu}+k_{j}^{\mu},
$$

where all indices are mod $n$ for an $n$-gluon amplitude. The invariant mass of this vector is $s_{i \cdots j}=K_{i \cdots j}^{2}$. Special cases include the two- and three-particle invariant masses, which are denoted by

$$
s_{i j} \equiv K_{i, j}^{2} \equiv\left(k_{i}+k_{j}\right)^{2}=2 k_{i} \cdot k_{j}, \quad s_{i j k} \equiv\left(k_{i}+k_{j}+k_{k}\right)^{2} .
$$

In color-ordered amplitudes only invariants with cyclicly-consecutive arguments need appear, e.g. $s_{i, i+1}$ and $s_{i, i+1, i+2}$. We also write, for the sum of massless momenta belonging to a set $A$,

$$
K_{A}^{\mu} \equiv \sum_{a_{i} \in A} k_{a_{i}}^{\mu}
$$

Spinor strings, such as

$$
\left\langle i^{+}\left|K_{A}\right| j^{+}\right\rangle=\sum_{a \in A}[i a]\langle a j\rangle, \quad\left\langle i^{-}\left|K_{A}\right| j^{-}\right\rangle=\sum_{a \in A}\langle i a\rangle[a j]
$$

and

$$
\begin{aligned}
\left\langle i^{-}|(a+b)| j^{-}\right\rangle & =\langle i a\rangle[a j]+\langle i b\rangle[b j], \\
\left\langle i^{+}|(a+b)(c+d)| j^{-}\right\rangle & =[i a]\left\langle a^{-}|(c+d)| j^{-}\right\rangle+[i b]\left\langle b^{-}|(c+d)| j^{-}\right\rangle,
\end{aligned}
$$

will also make appearances.

For one-loop amplitudes, the color decomposition is similar to the tree-level case (1) [43]. When all internal particles transform in the adjoint representation of $\mathrm{SU}\left(N_{c}\right)$, as is the case for $\mathcal{N}=4$ supersymmetric Yang-Mills theory, we have

$$
\mathcal{A}_{n}^{1-\text { loop }}\left(\left\{k_{i}, h_{i}, a_{i}\right\}\right)=\sum_{c=1}^{\lfloor n / 2\rfloor+1} \sum_{\sigma \in S_{n} / S_{n ; c}} \operatorname{Gr}_{n ; c}(\sigma) A_{n ; c}(\sigma),
$$


where $\lfloor x\rfloor$ is the largest integer less than or equal to $x$. The leading color-structure factor

$$
\operatorname{Gr}_{n ; 1}(1)=N_{c} \operatorname{Tr}\left(T^{a_{1}} \cdots T^{a_{n}}\right)
$$

is $N_{c}$ times the tree color factor. The subleading color structures are given by

$$
\operatorname{Gr}_{n ; c}(1)=\operatorname{Tr}\left(T^{a_{1}} \cdots T^{a_{c-1}}\right) \operatorname{Tr}\left(T^{a_{c}} \cdots T^{a_{n}}\right) .
$$

$S_{n}$ is the set of all permutations of $n$ objects, and $S_{n ; c}$ is the subset leaving $\mathrm{Gr}_{n ; c}$ invariant.

The one-loop subleading-color partial amplitudes are given by a sum over permutations of the leading-color ones [26]. Therefore we need to compute directly only the leading-color single-trace partial amplitudes $A_{n ; 1}\left(1^{h_{1}}, \ldots, n^{h_{n}}\right)$.

\section{REVIEW OF THE PROOF}

The proof of the BCFW recurrence relation [37] starts by introducing a parameterdependent shift of two of the external massless spinors, here labeled $j$ and $l$, in an $n$-point process,

$$
\begin{gathered}
\tilde{\lambda}_{j} \rightarrow \tilde{\lambda}_{j}-z \tilde{\lambda}_{l} \\
\lambda_{l} \rightarrow \lambda_{l}+z \lambda_{j}
\end{gathered}
$$

where $z$ is a complex number. This induces a shift of the corresponding momenta,

$$
\begin{aligned}
& p_{j}^{\mu} \rightarrow p_{j}^{\mu}(z)=p_{j}^{\mu}-\frac{z}{2}\left\langle j^{-}\left|\gamma^{\mu}\right| l^{-}\right\rangle, \\
& p_{l}^{\mu} \rightarrow p_{l}^{\mu}(z)=p_{l}^{\mu}+\frac{z}{2}\left\langle j^{-}\left|\gamma^{\mu}\right| l^{-}\right\rangle,
\end{aligned}
$$

which preserves their masslessness, $p_{j}^{2}(z)=0=p_{l}^{2}(z)$, as well as overall momentum conservation. This shift also implies,

$$
\begin{aligned}
& \not p_{j} \rightarrow \not p_{j}(z)=\not p_{j}-z\left(\left|l^{-}\right\rangle\left\langle j^{-}|+| j^{+}\right\rangle\left\langle l^{+}\right|\right), \\
& \not p_{l} \rightarrow \not p_{l}(z)=\not p_{l}+z\left(\left|l^{-}\right\rangle\left\langle j^{-}|+| j^{+}\right\rangle\left\langle l^{+}\right|\right) .
\end{aligned}
$$

Define

$$
A(z)=A\left(p_{1}, \ldots, p_{j}(z), p_{j+1}, \ldots, p_{l}(z), \ldots, p_{n}\right),
$$

which is an on-shell amplitude evaluated at a particular set of complex momenta. When $A$ is a tree amplitude or finite one-loop amplitude, $A(z)$ is a rational function of $z$. The physical amplitude is given by $A(0)$. 
Now consider the following quantity,

$$
\frac{1}{2 \pi i} \oint_{C} \frac{d z}{z} A(z)
$$

where the contour integral is taken around the circle at infinity. If $A(z) \rightarrow 0$ as $z \rightarrow \infty$, as in the tree-level cases considered by BCFW, then the integral vanishes, and we can evaluate this expression as follows,

$$
0=A(0)+\sum_{\text {poles } \alpha} \operatorname{Res}_{z=z_{\alpha}} \frac{A(z)}{z},
$$

where the sum is taken over the poles at the $z_{\alpha}$ of $A(z)$. We can then solve for $A(0)$,

$$
A(0)=-\sum_{\text {poles } \alpha} \operatorname{Res}_{z=z_{\alpha}} \frac{A(z)}{z} .
$$

If, on the other hand, $A(z) \rightarrow C_{\infty}$ as $z \rightarrow \infty$, the contour integral is equal to $C_{\infty}$, and this result is modified to,

$$
A(0)=C_{\infty}-\sum_{\text {poles }} \operatorname{Res}_{z=z_{\alpha}} \frac{A(z)}{z} .
$$

As explained in ref. [37], so long as $A(z)$ only has simple poles, each residue is given by factorizing the shifted amplitude on the appropriate pole in momentum invariants, so that at tree level,

$$
A(0)=C_{\infty}+\sum_{r, s, h} A_{L}^{h}\left(z=z_{r s}\right) \frac{i}{K_{r \cdots s}^{2}} A_{R}^{-h}\left(z=z_{r s}\right),
$$

where $h= \pm 1$ labels the helicity of the intermediate state. There is generically a double sum, labeled by $r, s$, over momentum poles, with legs $j$ and $l$ always appearing on opposite sides of the pole. The squared momentum associated with that pole, $K_{r \cdots s}^{2}$, is evaluated in the unshifted kinematics; whereas the on-shell amplitudes $A_{L}$ and $A_{R}$ are evaluated in kinematics that have been shifted by eq. (12), with $z=z_{r s}$. To extend the approach to one loop, the sum (20) must also be taken over the two ways of assigning the loop to the pair $\left(A_{L}, A_{R}\right)$.

The poles in $z$ arise from poles in momentum invariants; because of the structure of multiparticle factorization, in general only single poles arise. The same is almost true of collinear factorization at one loop; only lone powers of spinor products - and hence only single poles - appear in the splitting amplitudes in all helicity configurations except $(+++)$ and (-- ). In these identical-helicity cases, the tree splitting amplitude vanishes; however, the one-loop splitting amplitude has the form $[a b] /\langle a b\rangle^{2}$ or its conjugate [26], and double 
poles do arise. In no case does anything worse than a double pole arise. As we shall see in section $\mathrm{V}$, the double poles alter the form of the recurrence relation.

At tree level, it is always possible to find choices of reference momenta $j$ and $l$ such that there is no boundary term; and only single poles arise. For the finite one-loop amplitudes we will consider explicitly in this paper, life is not quite so simple, and we must confront each of these problems in turn.

\section{ALL-POSITIVE HELICITY ONE-LOOP AMPLITUDES}

Let us begin with the all-plus amplitude, for which Chalmers and the authors [38, 39] wrote a collinear-based conjecture [53],

$$
A_{n ; 1}=\frac{i N_{p}}{96 \pi^{2}} \frac{H_{n}}{\langle 12\rangle\langle 23\rangle \cdots\langle(n-1) n\rangle\langle n 1\rangle},
$$

where

$$
H_{n}=-\sum_{1 \leq i_{1}<i_{2}<i_{3}<i_{4} \leq n} \operatorname{Tr}_{-}\left[\not k_{i_{1}} \not k_{i_{2}} \not k_{i_{3}} \not k_{i_{4}}\right],
$$

and

$$
\begin{aligned}
\operatorname{Tr}_{-}\left[\not k_{i_{1}} \not k_{i_{2}} \not k_{i_{3}} \not k_{i_{4}}\right] & =\frac{1}{2} \operatorname{Tr}\left[\left(1-\gamma_{5}\right) \not k_{i_{1}} \not k_{i_{2}} \not k_{i_{3}} \not k_{i_{4}}\right] \\
& =\left\langle i_{1} i_{2}\right\rangle\left[i_{2} i_{3}\right]\left\langle i_{3} i_{4}\right\rangle\left[i_{4} i_{1}\right] .
\end{aligned}
$$

The conjecture was later proven by Mahlon [44]. The factor $N_{p}$ counts the number of states circulating in the loop, +1 or -1 for each adjoint representation bosonic or fermionic state,

respectively. For Dirac quarks in the fundamental representation, the contribution to $N_{p}$ is $-2 / N_{c}$, so that for QCD $N_{p}=2\left(1-n_{f} / N_{c}\right)$, where $n_{f}$ is the number of quark flavors. Because the corresponding tree-level amplitude vanishes for this helicity configuration, the one-loop amplitude (21) is finite, and is purely a rational function of the spinor products.

With a simple shift as given in eq. (12), it is easy to see that there must necessarily be a boundary term. Without loss of generality, we may take $l=n$. There are two cases to consider: when $j$ and $n$ are adjacent, and when they are not. Take the second case. Just as in the tree-level MHV case, two denominator factors are shifted,

$$
\langle(n-1) n\rangle \rightarrow\langle(n-1) n\rangle+z\langle(n-1) j\rangle, \quad\langle n 1\rangle \rightarrow\langle n 1\rangle+z\langle j 1\rangle
$$


This gives rise to two distinct single poles in $z$, a result that could have been obtained without knowledge of the answer, because the form of the denominator is fixed by general collinear- and multiparticle-factorization arguments.

Most terms in $H_{n}$ are either unshifted, or shifted by only a single power of $z$. These terms will fade away as $z \rightarrow \infty$. The only dangerous terms are those involving both $\not k_{j}$ and $k_{n}$. However,

$$
\frac{d}{d z} \not k_{j} k_{n}=0=\frac{d}{d z} \not k_{n} \not k_{j}
$$

so the terms proportional to $z^{2}$ are those with $i_{2}=j$ and $i_{4}=n$,

$$
\begin{aligned}
H_{n}^{\infty} & =z^{2}\left\langle j^{-}\left|K_{1 \cdots(j-1)}\right| n^{-}\right\rangle\left\langle j^{-}\left|K_{(j+1) \cdots(n-1)}\right| n^{-}\right\rangle \\
& =-z^{2}\left\langle j^{-}\left|K_{1 \cdots(j-1)}\right| n^{-}\right\rangle^{2} \\
& =-z^{2}\left\langle j^{-}\left|K_{(j+1) \cdots(n-1)}\right| n^{-}\right\rangle^{2} .
\end{aligned}
$$

Both the numerator and denominator scale as $z^{2}$, giving rise to a boundary term. In the other case, when $j$ and $n$ are adjacent (that is, $j=1$ or $n-1$ ), only one pole (one power of $z$ ) is generated from the denominator; but correspondingly, the numerator also scales as $z$, so that again we obtain a boundary term (whose form is somewhat more complicated, though).

It is not clear how to derive the boundary term without knowing the complete answer, so any recurrence relation we obtain with one is probably not useful for deriving the amplitude. We could, however, still use it for proving a conjecture.

Because tree-level amplitudes containing less than two negative-helicity gluons vanish, we know that the one-loop identical-helicity amplitude has no multi-particle poles, and that the only collinear singularities come from the tree-level $(-;++)$ splitting amplitude. This in turn implies that $A(z)$ in this case has only single poles, and accordingly we can write a recurrence relation for the all-plus amplitude,

$$
\begin{aligned}
A_{n}^{(1)}\left(1^{+}, \ldots, n^{+}\right)=C_{\infty}+ & \sum_{\substack{r=1 \\
(r, s) \neq(j, j),(1, n-n=\overline{1})}}^{j} \sum_{\substack{v=0 \\
h= \pm}}^{n-1} A_{s-r+2}^{(v)}\left(r^{+}, \ldots, \hat{\jmath}^{+}, \ldots, s^{+},-\hat{K}_{r \cdots s}^{h}\right) \frac{i}{K_{r \cdots s}^{2}} \\
& \times A_{n-s+r}^{(1-v)}\left(\hat{K}_{r \cdots s}^{-h},(s+1)^{+}, \ldots, \hat{n}^{+}, \ldots,(r-1)^{+}\right)
\end{aligned}
$$

(where $j \neq 1, n-1)$. For convenience we define $A_{n}^{(0)}=A_{n}^{\text {tree }}$ and $A_{n}^{(1)}=A_{n ; 1}^{1 \text {-loop }}$. The hatted 
momenta are evaluated using the shift (12), with

$$
z=z_{r s}=\frac{K_{r \cdots s}^{2}}{\left\langle j^{-}\left|K_{r \cdots s}\right| n^{-}\right\rangle} .
$$

Using the observation that tree amplitudes with a lone negative helicity continue to vanish generically for $n>3$, even when evaluated at complex momenta [23], only four terms survive in the sum,

$$
\begin{aligned}
& \frac{i}{K_{j, j+1}^{2}} A_{3}^{(0)}\left(\hat{\jmath}^{+},(j+1)^{+},-\hat{K}_{j, j+1}^{-}\right) A_{n-1}^{(1)}\left(\hat{K}_{j, j+1}^{+},(j+2)^{+}, \ldots, \hat{n}^{+}, \ldots,(j-1)^{+}\right) \\
& +\frac{i}{K_{j-1, j}^{2}} A_{3}^{(0)}\left((j-1)^{+}, \hat{\jmath}^{+},-\hat{K}_{j-1, j}^{-}\right) A_{n-1}^{(1)}\left(\hat{K}_{j-1, j}^{+},(j+1)^{+}, \ldots, \hat{n}^{+}, \ldots,(j-2)^{+}\right) \\
& +\frac{i}{K_{n-1, n}^{2}} A_{n-1}^{(1)}\left(1^{+}, \ldots, \hat{\jmath}^{+}, \ldots,(n-2)^{+}, \hat{K}_{n-1, n}^{+}\right) A_{3}^{(0)}\left(-\hat{K}_{n-1, n}^{-},(n-1)^{+}, \hat{n}^{+}\right) \\
& +\frac{i}{K_{n, 1}^{2}} A_{n-1}^{(1)}\left(2^{+}, \ldots, \hat{\jmath}^{+}, \ldots,(n-1)^{+}, \hat{K}_{n, 1}^{+}\right) A_{3}^{(0)}\left(-\hat{K}_{n, 1}^{-}, \hat{n}^{+}, 1^{+}\right) .
\end{aligned}
$$

Here the three-point amplitude, before permuting its arguments and shifting its momenta, is given by

$$
A_{3}^{(0)}\left(1^{+}, 2^{+}, 3^{-}\right)=-i \frac{[12]^{3}}{[23][31]} .
$$

With the choice of shift we have made, the three-point amplitudes $A^{(0)}\left(\hat{\jmath}^{+},(j+1)^{+},-\hat{K}_{j, j+1}^{-}\right)$ and $A^{(0)}\left((j-1)^{+}, \hat{\jmath}^{+},-\hat{K}_{j-1, j}^{-}\right)$vanish [35], so that the final relation takes the form,

$$
\begin{aligned}
A_{n}^{(1)}\left(1^{+}, \ldots,\right. & \left.n^{+}\right)=-\frac{i N_{p}}{96 \pi^{2}} \frac{\left\langle j^{-}\left|K_{(j+1) \cdots(n-1)}\right| n^{-}\right\rangle^{2}}{\langle 12\rangle\langle 23\rangle \cdots\langle(n-2)(n-1)\rangle\langle(n-1) j\rangle\langle j 1\rangle} \\
& +\frac{i}{K_{n-1, n}^{2}} A_{n-1}^{(1)}\left(1^{+}, \ldots, \hat{\jmath}^{+}, \ldots,(n-2)^{+}, \hat{K}_{n-1, n}^{+}\right) A_{3}^{(0)}\left(-\hat{K}_{n-1, n}^{-},(n-1)^{+}, \hat{n}^{+}\right) \\
& +\frac{i}{K_{n, 1}^{2}} A_{n-1}^{(1)}\left(2^{+}, \ldots, \hat{\jmath}^{+}, \ldots,(n-1)^{+}, \hat{K}_{n, 1}^{+}\right) A_{3}^{(0)}\left(-\hat{K}_{n, 1}^{-}, \hat{n}^{+}, 1^{+}\right),
\end{aligned}
$$

where $z=-\langle(n-1) n\rangle /\langle(n-1) j\rangle$ and $z=-\langle 1 n\rangle /\langle 1 j\rangle$ in the last two lines, respectively. We have checked this relation numerically through $n=15$, and find that it is indeed satisfied.

Can we find a recurrence which eliminates the boundary term? This is indeed possible, so long as we choose a more general shift of the original spinors. In particular, consider the following shift of the spinors $(j<l<n)$,

$$
\begin{aligned}
& \tilde{\lambda}_{j} \rightarrow \tilde{\lambda}_{j}-z \tilde{\lambda}_{l}-z \frac{\langle n j\rangle}{\langle l j\rangle} \tilde{\lambda}_{n}, \\
& \lambda_{l} \rightarrow \lambda_{l}+z \lambda_{j}, \\
& \lambda_{n} \rightarrow \lambda_{n}+z \frac{\langle n j\rangle}{\langle l j\rangle} \lambda_{j},
\end{aligned}
$$



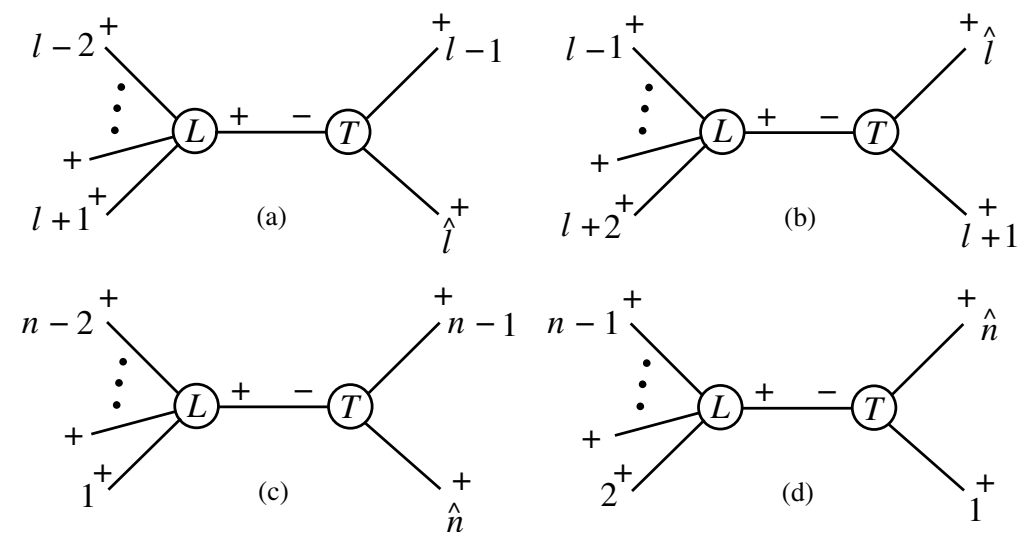

FIG. 1: Terms in the recursive expression (33) for $A_{n}^{(1)}\left(1^{+}, 2^{+}, \ldots, n^{+}\right)$. The vertices labeled by a $T$ are trees, and the ones labeled by an $L$ are loops.

which also keeps them massless and conserves overall four-momentum. The ratio $\langle n j\rangle /\langle l j\rangle$ allows $z$ to be assigned a definite weight under spinor phase rotations. So long as we choose $l$ not adjacent to $n$, and $j$ adjacent to at most one of $l$ and $n$, then we find that $A(z) \rightarrow 0$ as $z \rightarrow \infty$, and we obtain a recurrence free of surface terms. (We must accordingly take $n \geq 5$.) The price is that more terms must be included. For $1<j<l-1$ and $l<n-1$ (which requires $n \geq 6$ ), we obtain,

$$
\begin{aligned}
& A_{n}^{(1)}\left(1^{+}, \ldots, n^{+}\right)= \\
& \quad \frac{i}{K_{l-1, l}^{2}} A_{n-1}^{(1)}\left((l+1)^{+}, \ldots, \hat{n}^{+}, 1^{+}, \ldots, \hat{\jmath}^{+}, \ldots,(l-2)^{+}, \hat{K}_{l-1, l}^{+}\right) A_{3}^{(0)}\left(-\hat{K}_{l-1, l}^{-},(l-1)^{+}, \hat{l}^{+}\right) \\
& \quad+\frac{i}{K_{l, l+1}^{2}} A_{n-1}^{(1)}\left((l+2)^{+}, \ldots, \hat{n}^{+}, 1^{+}, \ldots, \hat{\jmath}^{+}, \ldots,(l-1)^{+}, \hat{K}_{l, l+1}^{+}\right) A_{3}^{(0)}\left(-\hat{K}_{l, l+1}^{-}, \hat{l}^{+},(l+1)^{+}\right) \\
& \quad+\frac{i}{K_{n-1, n}^{2}} A_{n-1}^{(1)}\left(1^{+}, \ldots, \hat{\jmath}^{+}, \ldots, \hat{l}^{+}, \ldots,(n-2)^{+}, \hat{K}_{n-1, n}^{+}\right) A_{3}^{(0)}\left(-\hat{K}_{n-1, n}^{-},(n-1)^{+}, \hat{n}^{+}\right) \\
& \quad+\frac{i}{K_{n, 1}^{2}} A_{n-1}^{(1)}\left(2^{+}, \ldots, \hat{\jmath}^{+}, \ldots, \hat{l}^{+}, \ldots,(n-1)^{+}, \hat{K}_{n, 1}^{+}\right) A_{3}^{(0)}\left(-\hat{K}_{n, 1}^{-}, \hat{n}^{+}, 1^{+}\right) .
\end{aligned}
$$

The respective values of the shift variable $z$ in the four terms in eq. (33) are

$$
-\frac{\langle(l-1) l\rangle}{\langle(l-1) j\rangle}, \quad-\frac{\langle(l+1) l\rangle}{\langle(l+1) j\rangle}, \quad-\frac{\langle l j\rangle}{\langle n j\rangle} \frac{\langle(n-1) n\rangle}{\langle(n-1) j\rangle}, \quad-\frac{\langle l j\rangle}{\langle n j\rangle} \frac{\langle 1 n\rangle}{\langle 1 j\rangle} .
$$

The recurrence relation (33) is depicted diagrammatically in fig. 1 . For $n=5$, there is an analogous relation containing only three terms. We have also checked the relation (33) numerically through $n=15$. 


\section{ONE-LOOP AMPLITUDES WITH A SINGLE NEGATIVE HELICITY}

Let us consider next $n$-gluon QCD amplitudes with a single negative-helicity gluon, and the remainder of positive helicity. These amplitudes were computed by Mahlon for an arbitrary number of external legs [44], using a loop-level generalization of the Berends-Giele recurrence relations [9]. In this section, we wish to reproduce these amplitudes, but in a simpler form, following instead the BCFW recursive methodology. As we shall see, the essential difference between the tree amplitudes and the one-negative-helicity loop amplitudes is the appearance of double poles. As a result of their appearance, a straightforward application of the BCFW recurrence misses single pole terms underlying the double poles. We will find a modification which can be written, for the case at hand, in terms of the soft functions which control the universal behavior of amplitudes as momenta vanish. In general, boundary terms may arise, but they can be avoided by an appropriate choice of a pair of legs $(j, l)$ to shift.

It is instructive to first inspect the analytic properties of the five-point amplitude, as its features will serve as a guide to finding an all- $n$ recurrence relation. The one-loop five-point amplitude with a single negative helicity leg was first calculated using string-based methods and is given by $[38,45]$,

$$
A_{5}^{(1)}\left(1^{-}, 2^{+}, 3^{+}, 4^{+}, 5^{+}\right)=i \frac{N_{p}}{96 \pi^{2}} \frac{1}{\langle 34\rangle^{2}}\left[-\frac{[25]^{3}}{[12][51]}+\frac{\langle 14\rangle^{3}[45]\langle 35\rangle}{\langle 12\rangle\langle 23\rangle\langle 45\rangle^{2}}-\frac{\langle 13\rangle^{3}[32]\langle 42\rangle}{\langle 15\rangle\langle 54\rangle\langle 32\rangle^{2}}\right] .
$$

Now consider the effect of the shift,

$$
\begin{aligned}
& \lambda_{1} \rightarrow \lambda_{1}, \\
& \tilde{\lambda}_{1} \rightarrow \tilde{\lambda}_{1}-z \tilde{\lambda}_{2}, \\
& \lambda_{2} \rightarrow \lambda_{2}+z \lambda_{1}, \\
& \tilde{\lambda}_{2} \rightarrow \tilde{\lambda}_{2} .
\end{aligned}
$$

Applying it to eq. (35) gives the shifted amplitude,

$$
\begin{aligned}
A_{5}^{(1)}(z)=i \frac{N_{p}}{96 \pi^{2}} \frac{1}{\langle 34\rangle^{2}}[ & -\frac{[25]^{3}}{[12]([51]-z[52])}+\frac{\langle 14\rangle^{3}[45]\langle 35\rangle}{\langle 12\rangle(\langle 23\rangle+z\langle 13\rangle)\langle 45\rangle^{2}} \\
& \left.-\frac{\langle 13\rangle^{3}[32](\langle 42\rangle+z\langle 41\rangle)}{\langle 15\rangle\langle 54\rangle(\langle 32\rangle+z\langle 31\rangle)^{2}}\right] .
\end{aligned}
$$

Since $A_{5}^{(1)}(z)$ vanishes as $z \rightarrow \infty$, the choice of shift (36) does not generate a boundary term at $z \rightarrow \infty$. Other shifts are also possible, but not all are as useful; some do generate 
boundary terms. Our shifted amplitude (37) has a new feature: the appearance of a double pole in $z$. The double pole implies that the BCFW recurrence relation cannot be applied directly, because it relied on the amplitude having only single poles, as is the case at tree level. Nevertheless, we will use eq. (37) to track down the required modifications.

To proceed with our investigation, rewrite eq. (37) as a sum over pole terms,

$$
\begin{aligned}
A_{5}^{(1)}(z)=i \frac{N_{p}}{96 \pi^{2}} \frac{1}{\langle 34\rangle^{2}}[- & \frac{[25]^{3}}{[12]([51]-z[52])}+\frac{\langle 14\rangle^{3}[45]\langle 35\rangle}{\langle 12\rangle(\langle 23\rangle+z\langle 13\rangle)\langle 45\rangle^{2}} \\
& -\frac{\langle 13\rangle^{3}[32]\langle 43\rangle\langle 21\rangle}{\langle 15\rangle\langle 54\rangle\langle 31\rangle(\langle 32\rangle+z\langle 31\rangle)^{2}} \\
& \left.-\frac{\langle 13\rangle^{3}[32]\langle 41\rangle}{\langle 15\rangle\langle 54\rangle\langle 31\rangle(\langle 32\rangle+z\langle 31\rangle)}\right]
\end{aligned}
$$

where we have used the Schouten identity to expose the single pole sitting under the double pole. Taking $z=0$, we obtain a form of the amplitude whose terms we wish to map onto terms of a recurrence relation,

$$
\begin{aligned}
A_{5}^{(1)}(z=0)=i \frac{N_{p}}{96 \pi^{2}} & {\left[-\frac{[25]^{3}}{\langle 34\rangle^{2}[12][51]}+\frac{\langle 14\rangle^{3}[45]\langle 35\rangle}{\langle 34\rangle^{2}\langle 12\rangle\langle 23\rangle\langle 45\rangle^{2}}\right.} \\
& \left.+\frac{\langle 13\rangle^{2}[32]\langle 21\rangle}{\langle 34\rangle\langle 15\rangle\langle 45\rangle\langle 32\rangle^{2}}+\frac{\langle 13\rangle^{2}[32]\langle 41\rangle}{\langle 34\rangle^{2}\langle 15\rangle\langle 54\rangle\langle 32\rangle}\right] .
\end{aligned}
$$

The appearance of a double pole in eq. (38) may seem puzzling at first sight. It can be understood from the structure of the one-loop three-vertex with identical helicities, used for obtaining one-loop splitting amplitudes [26]. When the momenta of legs 1 and 2 become collinear, the vertex becomes $[46,47]$

$$
\begin{aligned}
A_{3}^{(1)}\left(1^{+}, 2^{+}, 3^{+}\right) & =-i \frac{N_{p}}{96 \pi^{2}} \frac{\varepsilon_{3}^{(+)} \cdot\left(k_{1}-k_{2}\right)}{\sqrt{2}}\left[\varepsilon_{1}^{(+)} \cdot \varepsilon_{2}^{(+)}-2 \frac{k_{1} \cdot \varepsilon_{2}^{(+)} k_{2} \cdot \varepsilon_{1}^{(+)}}{K_{12}^{2}}\right] \\
& =-i \frac{N_{p}}{96 \pi^{2}} \frac{[12][23][31]}{K_{12}^{2}}
\end{aligned}
$$

It is useful to expose the kinematic pole, so we define

$$
A_{3}^{(1)}\left(1^{+}, 2^{+}, 3^{+}\right) \equiv \frac{1}{K_{12}^{2}} V_{3}^{(1)}\left(1^{+}, 2^{+}, 3^{+}\right) .
$$

The propagator of the factorized leg provides a second factor of $1 / K_{12}^{2}$, leading to the observed double poles in the amplitude. These poles make their explicit appearance in eq. (35) as spinor products $\langle 23\rangle,\langle 34\rangle$ and $\langle 45\rangle$ appearing quadratically in the denominator. (They can also be understood as arising from those collinear singularities, with $k_{a}$ parallel 

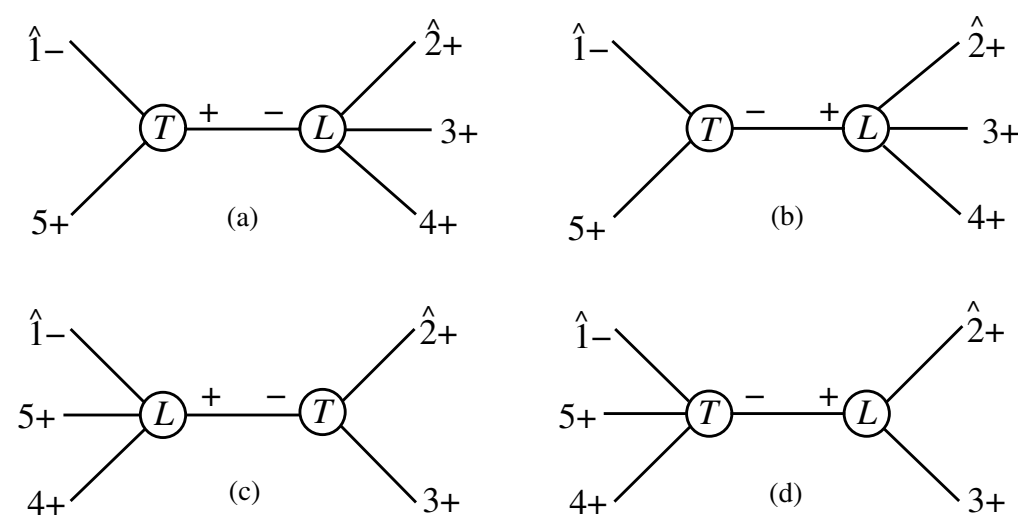

FIG. 2: Terms in the recursive expression for $A_{5}^{(1)}\left(1^{-}, 2^{+}, 3^{+}, 4^{+}, 5^{+}\right)$.

to $k_{b}$, and $k_{a} \approx x\left(k_{a}+k_{b}\right)$, which involve the one-loop splitting amplitude denoted by [26] $\left.\operatorname{Split}_{+}^{1-\text { loop }}\left(x, a^{+}, b^{+}\right)=-i N_{p} /\left(96 \pi^{2}\right) \times \sqrt{x(1-x)}[a b] /\langle a b\rangle^{2}.\right)$

We turn now to the recursive construction of the five-point amplitude. Our input expressions are the one-loop vertex (41) as well as the one-loop four-point amplitudes [48],

$$
\begin{aligned}
& A_{4}^{(1)}\left(1^{+}, 2^{+}, 3^{+}, 4^{+}\right)=-i \frac{N_{p}}{96 \pi^{2}} \frac{[12][34]}{\langle 12\rangle\langle 34\rangle} \\
& A_{4}^{(1)}\left(1^{-}, 2^{+}, 3^{+}, 4^{+}\right)=i \frac{N_{p}}{96 \pi^{2}} \frac{\langle 24\rangle[24]^{3}}{[12]\langle 23\rangle\langle 34\rangle[41]} .
\end{aligned}
$$

Although the symmetry is not manifest in the form we have written it, $A_{4}^{(1)}\left(1^{+}, 2^{+}, 3^{+}, 4^{+}\right)$is invariant under cyclic permutations of its arguments. We shall also need the MHV $n$-gluon tree amplitudes [7-9],

$$
A_{n}^{(0)}\left(1^{+}, \ldots,(j-1)^{+}, j^{-},(j+1)^{+}, \ldots,(k-1)^{+}, k^{-},(k+1)^{+}, \ldots, n^{+}\right)=i \frac{\langle j k\rangle^{4}}{\langle 12\rangle\langle 23\rangle \cdots\langle n 1\rangle},
$$

and the three-point amplitude for $(++-)$, given in eq. (30).

The proof of the recurrence relations in ref. [37] leads us to expect a recurrence relation for the five-point one-loop amplitude of the form,

$$
\begin{aligned}
A_{5}^{(1)}\left(1^{-}, 2^{+}, 3^{+}, 4^{+}, 5^{+}\right) \sim & A_{3}^{(0)}\left(\hat{1}^{-},-\hat{K}_{15}^{+}, 5^{+}\right) \frac{i}{K_{15}^{2}} A_{4}^{(1)}\left(\hat{2}^{+}, 3^{+}, 4^{+}, \hat{K}_{15}^{-}\right) \\
& +A_{3}^{(0)}\left(\hat{1}^{-},-\hat{K}_{15}^{-}, 5^{+}\right) \frac{i}{K_{15}^{2}} A_{4}^{(1)}\left(\hat{2}^{+}, 3^{+}, 4^{+}, \hat{K}_{15}^{+}\right) \\
& +A_{4}^{(1)}\left(\hat{1}^{-}, \hat{K}_{23}^{+}, 4^{+}, 5^{+}\right) \frac{i}{K_{23}^{2}} A_{3}^{(0)}\left(\hat{2}^{+}, 3^{+},-\hat{K}_{23}^{-}\right) \\
& +A_{4}^{(0)}\left(\hat{1}^{-}, \hat{K}_{23}^{-}, 4^{+}, 5^{+}\right) \frac{i}{\left(K_{23}^{2}\right)^{2}} V_{3}^{(1)}\left(\hat{2}^{+}, 3^{+},-\hat{K}_{23}^{+}\right),
\end{aligned}
$$


corresponding to the four diagrams illustrated in fig. 2. Following the discussion of section III, it is straightforward to evaluate each term. As we shall see, however, these terms do not yield the complete answer, because they miss a single pole lying underneath the double pole of the last term. However, an inspection of the discrepancy will allow us to determine an $n$-point recurrence relation.

The first diagram, shown in fig. 2(a), corresponding to the first term in eq. (45), simply vanishes,

$$
D^{(a)}=A_{3}^{(0)}\left(\hat{1}^{-},-\hat{K}_{15}^{+}, 5^{+}\right) \frac{i}{K_{15}^{2}} A_{4}^{(1)}\left(\hat{2}^{+}, 3^{+}, 4^{+}, \hat{K}_{15}^{-}\right)=0,
$$

because [35]

$$
A_{3}^{(0)}\left(\hat{1}^{-},-\hat{K}_{15}^{+}, 5^{+}\right) \propto\left[\left(-\hat{K}_{15}\right) 5\right]^{3} \propto\left\langle 1^{-}|(1+5)| 5^{-}\right\rangle^{3}=0 .
$$

We may evaluate the next diagram, shown in fig. 2(b), corresponding to the second term in eq. (45), to obtain,

$$
\begin{aligned}
D^{(b)} & =A_{3}^{(0)}\left(\hat{1}^{-},-\hat{K}_{15}^{-}, 5^{+}\right) \frac{i}{K_{15}^{2}} A_{4}^{(1)}\left(\hat{2}^{+}, 3^{+}, 4^{+}, \hat{K}_{15}^{+}\right) \\
& =i \frac{N_{p}}{96 \pi^{2}} \frac{\left\langle\hat{1}\left(-\hat{K}_{15}\right)\right\rangle^{3}}{\langle\hat{1} 5\rangle\left\langle 5\left(-\hat{K}_{15}\right)\right\rangle} \times \frac{1}{K_{15}^{2}} \times \frac{[34]\left[\hat{2} \hat{K}_{15}\right]}{\langle 34\rangle\left\langle\hat{2} \hat{K}_{15}\right\rangle} \\
& =i \frac{N_{p}}{96 \pi^{2}} \frac{\left\langle 1^{-}\left|K_{15}\right| 2^{-}\right\rangle^{3}}{\omega \bar{\omega}\langle 15\rangle\left\langle 5^{-}\left|K_{15}\right| 2^{-}\right\rangle} \times \frac{1}{K_{15}^{2}} \times \frac{[34]\left\langle 1^{-}\left|K_{15}\right| 2^{-}\right\rangle}{\langle 34\rangle\left\langle\hat{2}^{-}\left|K_{15}\right| 2^{-}\right\rangle} .
\end{aligned}
$$

where $\omega=\left[\hat{K}_{15} 2\right]$ and $\bar{\omega}=\left\langle 1 \hat{K}_{15}\right\rangle$. Using

$$
\left\langle\hat{2}^{-}\left|K_{15}\right| 2^{-}\right\rangle=\left\langle 2^{-}|(1+5)| 2^{-}\right\rangle+\frac{K_{15}^{2}}{\left\langle 1^{-}|(1+5)| 2^{-}\right\rangle}\left\langle 1^{-}|(1+5)| 2^{-}\right\rangle=K_{34}^{2},
$$

and

$$
\omega \bar{\omega}=\left\langle 1^{-}\left|K_{15}\right| 2^{-}\right\rangle=\langle 15\rangle[52],
$$

we have that diagram (b) of fig. 2 simplifies to

$$
D^{(b)}=-i \frac{N_{p}}{96 \pi^{2}} \frac{[25]^{3}}{\langle 34\rangle^{2}[12][51]} .
$$

This matches precisely the first term in our target expression (39).

Similarly, it is not difficult to work out the diagram shown in fig. 2(c), with the result

$$
\begin{aligned}
D^{(c)} & =A_{4}^{(0)}\left(\hat{1}^{-}, \hat{K}_{23}^{+}, 4^{+}, 5^{+}\right) \frac{i}{K_{23}^{2}} A_{3}^{(0)}\left(\hat{2}^{+}, 3^{+},-\hat{K}_{23}^{-}\right) \\
& =i \frac{N_{p}}{96 \pi^{2}} \frac{\langle 14\rangle^{3}[45]\langle 35\rangle}{\langle 34\rangle^{2}\langle 12\rangle\langle 23\rangle\langle 45\rangle^{2}}
\end{aligned}
$$


matching the second term of eq. (39). Finally, for diagram (d) we obtain the double-pole contribution very easily,

$$
\begin{aligned}
D_{2}^{(d)} & =A_{4}^{(0)}\left(\hat{1}^{-}, \hat{K}_{23}^{-}, 4^{+}, 5^{+}\right) \frac{i}{\left(K_{23}^{2}\right)^{2}} V_{3}^{(1)}\left(\hat{2}^{+}, 3^{+},-\hat{K}_{23}^{+}\right) \\
& =i \frac{N_{p}}{96 \pi^{2}} \frac{\langle 13\rangle^{2}[32]\langle 21\rangle}{\langle 34\rangle\langle 15\rangle\langle 45\rangle\langle 32\rangle^{2}}
\end{aligned}
$$

matching the third term of the target expression (39).

We now must confront the question of constructing the single-pole contribution in diagram (d) which underlies the double pole. One might suspect that the single-pole terms are given by series expanding the amplitudes in momenta around the pole. This suggests that we need the behavior of the amplitudes on both sides of the double pole as $\hat{K}_{23}$ vanishes. The functional behavior of amplitudes in this limit is controlled by universal soft functions, so it seems reasonable that these functions will appear in the term we wish to construct. With a little experimentation, it is not difficult to find a candidate single-pole term given by

$$
D_{1}^{(d)}=A_{4}^{(0)}\left(\hat{1}^{-}, \hat{K}_{23}^{-}, 4^{+}, 5^{+}\right) \mathcal{S}^{(0)}\left(\hat{1}, \hat{K}_{23}^{-}, 4\right) \frac{i}{K_{23}^{2}} \mathcal{S}^{(0)}\left(3,-\hat{K}_{23}^{+}, \hat{2}\right) V_{3}^{(1)}\left(\hat{2}^{+}, 3^{+},-\hat{K}_{23}^{+}\right)
$$

where the soft factors depend only on the helicity of soft gluon and are given by [41]

$$
\begin{aligned}
\mathcal{S}^{(0)}\left(a, s^{+}, b\right) & =\frac{\langle a b\rangle}{\langle a s\rangle\langle s b\rangle}, \\
\mathcal{S}^{(0)}\left(a, s^{-}, b\right) & =-\frac{[a b]}{[a s][s b]} .
\end{aligned}
$$

Evaluating eq. (54) we have

$$
D_{1}^{(d)}=i \frac{N_{p}}{96 \pi^{2}} \frac{\langle 13\rangle^{2}[32]\langle 41\rangle}{\langle 3\rangle^{2}\langle 15\rangle\langle 54\rangle\langle 32\rangle}
$$

so it reproduces the last term in the amplitude (39).

We now take the value of diagram of fig. 2(d) to be the sum of the single- and double-pole contributions,

$$
\begin{aligned}
D^{(d)} & =D_{1}^{(d)}+D_{2}^{(d)} \\
& =i \frac{N_{p}}{96 \pi^{2}}\left[\frac{\langle 13\rangle^{2}[32]\langle 21\rangle}{\langle 34\rangle\langle 15\rangle\langle 45\rangle\langle 32\rangle^{2}}+\frac{\langle 13\rangle^{2}[32]\langle 41\rangle}{\langle 34\rangle^{2}\langle 15\rangle\langle 54\rangle\langle 32\rangle}\right],
\end{aligned}
$$

which matches the last two terms in the amplitude (39). 

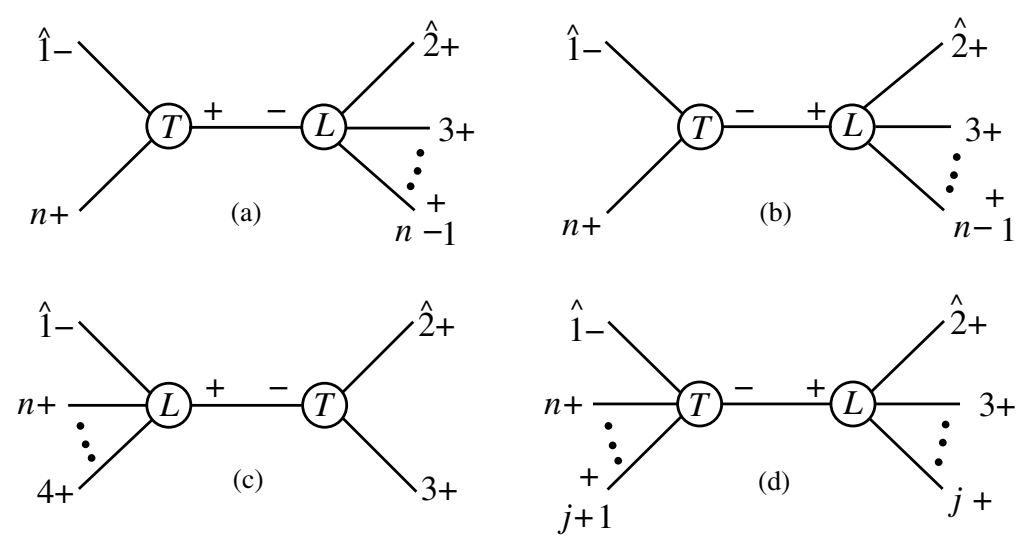

FIG. 3: The diagrams describing a recursive formula for $A_{n}^{(1)}\left(1^{-}, 2^{+}, \ldots, n^{+}\right)$. Diagram (a) vanishes, while diagram (d) needs to be summed over $j$. For $j=3$ diagram (d) contains both single-pole and double-pole contributions.

We have thus succeeded in finding a simple interpretation of the five-point amplitude (39) in terms of a recursive diagrammatic construction,

$$
A_{5}^{(1)}\left(1^{-}, 2^{+}, 3^{+}, 4^{+}, 5^{+}\right)=D^{(a)}+D^{(b)}+D^{(c)}+D^{(d)},
$$

corresponding to the diagrams in fig. 2, after interpreting diagram (d) as a sum over singlepole and double-pole contributions.

This result also makes clear what the generalization to $n$ legs ought to be. It is,

$$
\begin{aligned}
& A_{n}^{(1)}\left(1^{-}, 2^{+}, \ldots, n^{+}\right) \\
& =A_{n-1}^{(1)}\left(4^{+}, 5^{+}, \ldots, n^{+}, \hat{1}^{-},-\hat{K}_{23}^{+}\right) \frac{i}{K_{23}^{2}} A_{3}^{(0)}\left(\hat{2}^{+}, 3^{+}, \hat{K}_{23}^{-}\right) \\
& +\sum_{j=4}^{n-1} A_{n-j+2}^{(0)}\left((j+1)^{+}, 5^{+}, \ldots, n^{+}, \hat{1}^{-}, \hat{K}_{2 \ldots j}^{-}\right) \frac{i}{K_{2 \ldots j}^{2}} A_{j}^{(1)}\left(\hat{2}^{+}, 3^{+}, \ldots, j^{+},-\hat{K}_{2 \ldots j}^{+}\right) \\
& +A_{n-1}^{(0)}\left(4^{+}, 5^{+}, \ldots, n^{+}, \hat{1}^{-}, \hat{K}_{23}^{-}\right) \frac{i}{\left(K_{23}^{2}\right)^{2}} V_{3}^{(1)}\left(\hat{2}^{+}, 3^{+},-\hat{K}_{23}^{+}\right) \\
& \quad \times\left(1+K_{23}^{2} \mathcal{S}^{(0)}\left(\hat{1}, \hat{K}_{23}^{-}, 4\right) \mathcal{S}^{(0)}\left(3,-\hat{K}_{23}^{+}, \hat{2}\right)\right) .
\end{aligned}
$$

For self-consistency, amplitudes generated by this recurrence relation must vanish under the shift (36) in the limit $z \rightarrow \infty$. Otherwise, there would be a boundary term not included in the recurrence relation. To show that the amplitudes generated by eq. (60) satisfy this criterion, we first observe that under the shift the explicit poles appearing in the amplitude will behave either as $1 / z$ for the single poles or $1 / z^{2}$ for the $1 /\left(K_{23}^{2}\right)^{2}$ double pole. In fact, 
these are the only places $z$-dependence will arise. (Basically, the point of the recursive decomposition is to expose the $z$-dependence as pole factors multiplied by $z$-independent residues.) To see this explicitly, first note that $\lambda_{1}$ is unaffected by the shift, while $\tilde{\lambda}_{1}$ is affected. In the $j$ th term in eq. (60), consider the spinor product $[1 a]$ for an arbitrary leg $a$ (not equal to $\hat{K}_{2 \cdots j}^{-}$). It looks like it might develop $z$-dependence in the next shift. However, what actually appears is the hatted expression,

$$
\begin{aligned}
{[\hat{1} a] } & =[1 a]+\frac{K_{2 \cdots j}^{2}}{\left\langle 1^{-}\left|K_{2 \cdots j}\right| 2^{-}\right\rangle}[2 a]=\frac{\left\langle 2^{+}\left|K_{(j+1) \cdots 1} \not k_{1}\right| a^{-}\right\rangle-\left\langle 2^{+}\left|K_{(j+1) \cdots 1} \not K_{(j+1) \cdots 1}\right| a^{-}\right\rangle}{\left\langle 1^{-}\left|K_{(j+1) \cdots 1}\right| 2^{-}\right\rangle} \\
& =-\frac{\left\langle 2^{+}\left|K_{(j+1) \cdots 1} K_{(j+1) \cdots n}\right| a^{-}\right\rangle}{\left\langle 1^{-}\left|K_{(j+1) \cdots n}\right| 2^{-}\right\rangle}
\end{aligned}
$$

Since $\lambda_{1}$ and $\tilde{\lambda}_{2}$ are unaffected by the shift, the only possible source of $z$-dependence is from the $k_{1}$ inside $K_{2 \cdots j}$. But this term is proportional to [21], and thus unaffected (because the $\tilde{\lambda}_{1}$ shift is proportional to $\tilde{\lambda}_{2}$ ).

Similarly, the expressions on the other side of the $K_{2 \cdots j}^{2}$ pole which might appear to develop $z$-dependence are of the form

$$
\begin{aligned}
\langle\hat{2} b\rangle & =\langle 2 b\rangle-\frac{K_{2 \cdots j}^{2}}{\left\langle 1^{-}\left|K_{2 \cdots j}\right| b^{-}\right\rangle}\langle 1 b\rangle \\
& =-\frac{\left\langle 1^{-}\left|K_{2 \cdots j} K_{3 \cdots j}\right| b^{+}\right\rangle}{\left\langle 1^{-}\left|K_{2 \cdots j}\right| 2^{-}\right\rangle}
\end{aligned}
$$

and so they also are easily be seen to be unaffected by the subsequent $z$ shift. An expression involving the intermediate hatted momentum, like $\left\langle\hat{2} \hat{K}_{2 \cdots j}\right\rangle$, can be re-written as

$$
\left\langle\hat{2} \hat{K}_{2 \cdots j}\right\rangle=\frac{\left\langle\hat{2}^{-}\left|K_{2 \cdots j}\right| 2^{-}\right\rangle}{\left\langle 1^{-}\left|K_{2 \cdots j}\right| 2^{-}\right\rangle}
$$

and then $\mathbb{K}_{2 \cdots j}$ can be split into a sum of momenta in the numerator, applying eq. (62) to each term. The denominator is also inert. Thus the only $z$-dependence in eq. (60) is from the explicit $K_{2 \cdots j}^{2}$ pole factors, and the expression vanishes as $z \rightarrow \infty$.

We have checked the recurrence relation (60) numerically against the computation of Mahlon [44] through $n=15$. The relation leads straightforwardly to compact expressions for higher-point amplitudes. For example, the six-point amplitude can be written as,

$$
\begin{aligned}
& A_{6 ; 1}\left(1^{-}, 2^{+}, 3^{+}, 4^{+}, 5^{+}, 6^{+}\right) \\
& \quad=i \frac{N_{p}}{96 \pi^{2}}\left[\frac{\left\langle 1^{-}|(2+3)| 6^{-}\right\rangle^{3}}{\langle 12\rangle\langle 23\rangle\langle 45\rangle^{2} s_{123}\left\langle 3^{-}|(1+2)| 6^{-}\right\rangle}+\frac{\left\langle 1^{-}|(3+4)| 2^{-}\right\rangle^{3}}{\langle 34\rangle^{2}\langle 56\rangle\langle 61\rangle s_{234}\left\langle 5^{-}|(3+4)| 2^{-}\right\rangle}\right.
\end{aligned}
$$




$$
\begin{aligned}
& +\frac{[26]^{3}}{[12][61] s_{345}}\left(\frac{[23][34]}{\langle 45\rangle\left\langle 5^{-}|(3+4)| 2^{-}\right\rangle}-\frac{[45][56]}{\langle 34\rangle\left\langle 3^{-}|(1+2)| 6^{-}\right\rangle}+\frac{[35]}{\langle 34\rangle\langle 45\rangle}\right) \\
& -\frac{\langle 13\rangle^{3}[23]\langle 24\rangle}{\langle 23\rangle^{2}\langle 34\rangle^{2}\langle 45\rangle\langle 56\rangle\langle 61\rangle}+\frac{\langle 15\rangle^{3}\langle 46\rangle[56]}{\langle 12\rangle\langle 23\rangle\langle 34\rangle\langle 45\rangle^{2}\langle 56\rangle^{2}} \\
& \left.-\frac{\langle 14\rangle^{3}\langle 35\rangle\left\langle 1^{-}|(2+3)| 4^{-}\right\rangle}{\langle 12\rangle\langle 23\rangle\langle 34\rangle^{2}\langle 45\rangle^{2}\langle 56\rangle\langle 61\rangle}\right] .
\end{aligned}
$$

Similarly, in the seven-point case we obtain the form,

$$
A_{7 ; 1}\left(1^{-}, 2^{+}, 3^{+}, 4^{+}, 5^{+}, 6^{+}, 7^{+}\right)=\hat{A}_{7}^{-}+\left.\hat{A}_{7}^{-}\right|_{\text {flip }},
$$

where

$$
\left.X(1,2,3,4,5,6,7)\right|_{\text {flip }}=-X(1,7,6,5,4,3,2),
$$

and

$$
\begin{aligned}
& \hat{A}_{7}^{-}=i \frac{N_{p}}{96 \pi^{2}}\left[\frac{\left\langle 1^{-}|(2+3)| 7^{-}\right\rangle^{3}}{\langle 12\rangle\langle 23\rangle\langle 45\rangle\langle 56\rangle s_{123} s_{456}\left\langle 3^{-}|(1+2)| 7^{-}\right\rangle}\right. \\
& \times\left(\frac{s_{45}\left\langle 1^{-}|(2+3)| 4^{-}\right\rangle}{\left\langle 1^{-}|(2+3)(4+5)| 6^{+}\right\rangle}+\frac{\left\langle 6^{+}|(4+5)(5+6)| 7^{-}\right\rangle}{\left\langle 4^{-}|(5+6)| 7^{-}\right\rangle}\right) \\
&+\frac{\left\langle 1^{-}|(5+6)| 7^{-}\right\rangle^{3}}{\langle 12\rangle\langle 23\rangle\langle 34\rangle\langle 56\rangle^{2} s_{567}\left\langle 4^{-}|(5+6)| 7^{-}\right\rangle} \\
&+\frac{[27]^{3}}{[12]\langle 34\rangle\langle 45\rangle\langle 56\rangle[71] s_{712}}\left(\frac{1}{2}[36]+\frac{\left\langle 6^{+}|(4+5)(5+6)| 7^{-}\right\rangle}{\left\langle 3^{-}|(1+2)| 7^{-}\right\rangle}\right. \\
&-\frac{\left.\langle 13\rangle^{3}[23]\langle 24\rangle \quad-\frac{s_{45}}{2} \frac{\left\langle 2^{+}|(3+4)(5+6)| 7^{-}\right\rangle}{\left\langle 233^{-}|(1+2)| 7^{-}\right\rangle\left\langle 6^{-}|(7+1)| 2^{-}\right\rangle}\right)}{\langle 234\rangle^{2}\langle 45\rangle\langle 56\rangle\langle 67\rangle\langle 71\rangle}-\frac{\langle 14\rangle^{3}\langle 35\rangle\left\langle 1^{-}|(2+3)| 4^{-}\right\rangle}{\langle 12\rangle\langle 23\rangle\langle 34\rangle^{2}\langle 45\rangle^{2}\langle 56\rangle\langle 67\rangle\langle 71\rangle} \\
&\left.-\frac{1}{2} \frac{\left\langle 1^{-}|(2+3)(4+5)| 1^{+}\right\rangle^{3}}{\langle 12\rangle\langle 23\rangle\langle 45\rangle^{2}\langle 67\rangle\langle 71\rangle\left\langle 1^{-}|(6+7)(4+5)| 3^{+}\right\rangle\left\langle 1^{-}|(2+3)(4+5)| 6^{+}\right\rangle}\right] .
\end{aligned}
$$

Although the amplitudes (64) and (67) are quite compact in comparison with the expressions appearing in ref. [44], they do contain spurious denominators of the form $\left\langle a^{-}|(b+c)| d^{-}\right\rangle$and $\left\langle a^{-}|(b+c)(c+d)| e^{+}\right\rangle$. Such denominators typically arise from reducing pentagon integrals to lower-point integrals. It is of course no surprise that we generate such denominators, given the origin of the BCFW-type recurrences in the relations between coefficients of one-loop amplitudes. Similar spurious denominators also arise in the CSW construction of amplitudes from MHV vertices. In fact, it appears empirically that spurious 
denominators are necessary to obtain the most compact forms of amplitudes that contain many multi-particle factorization poles.

\section{CONCLUSIONS AND OUTLOOK}

In this paper, we presented a recursive approach to obtaining rational terms appearing in non-supersymmetric one-loop amplitudes, based on the Britto, Cachazo, Feng and Witten $[35,37]$ recursive approach to tree-level amplitudes. A striking feature of the proof of the method in ref. [37] is that it relies only on very general properties of field theory and complex function theory. It should therefore apply much more broadly than to massless gauge theory tree amplitudes. This motivated us to revisit the question of loop amplitudes.

In this paper, as an initial application, we focused on the finite one-loop all-gluon QCD amplitudes which are purely rational: amplitudes with all gluons of positive helicity, and those with a lone negative-helicity gluon. These amplitudes had been obtained previously in refs. [38, 39, 44], and are therefore quite useful as a laboratory for studying the new features appearing at loop level. Two essential differences arising at loop level are boundary terms and double poles, both of which modify the form of the recurrence. The boundary terms interfere with obtaining a useful recurrence relation, but via an appropriate choice of shifts we can avoid these. From general field theory considerations, tree-level amplitudes cannot have double poles in kinematic invariants. However, at loop level double poles do arise, when using complex momenta. These double poles induce extra terms in the recurrence relations, which appear to be related to the universal functions describing soft gluon emission, at least for the case discussed here.

In general, we envisage that the logarithmic parts of one-loop QCD amplitudes will be obtainable from the unitarity or related methods, while the rational function parts will be calculable using recursive methods. We can think of all-gluon QCD amplitudes as being composed of three distinct terms: an $\mathcal{N}=4$ supersymmetric amplitude; a multiple of the contribution of an $\mathcal{N}=1$ supersymmetric chiral multiplet; and a multiple of the contribution of a colored scalar circulating in the loop. (There is a similar type of decomposition for amplitudes with external fermions [49].) The first two terms can be computed from their four-dimensional cuts, for which in turn the computation of massless four-dimensional helicity amplitudes suffices. Many such amplitudes have already been computed, in both 
the $\mathcal{N}=4[21,22,24,26]$ and $\mathcal{N}=1$ cases $[27,50,51]$

The scalar term, which is non-supersymmetric, can also be computed via unitarity, but the computation would require tree amplitudes with the scalar propagating in the full $D=4-2 \epsilon$ spacetime [28], which are more cumbersome for large numbers of external legs. We can, however, think of splitting the scalar contribution up further into those terms which can be computed from the four-dimensional cuts, and a remainder. Indeed, the cut-containing parts of the scalar loop amplitudes with two negative-helicity gluons and $(n-2)$ of positive helicity have already been computed $[27,52]$. To the terms with discontinuities such as dilogarithms and logarithms, it is convenient to add certain rational terms which remove spurious (unphysical) singularities [45]. For example, the function $\ln \left[\left(-s_{1}\right) /\left(-s_{2}\right)\right] /\left(s_{1}-s_{2}\right)^{2}$ generically appears in one-loop amplitudes, and has a singularity as the momentum invariant $s_{1}$ approaches $s_{2}$, which is not associated with any physical factorization. This singularity can be removed by replacing

$$
\frac{\ln \left(\frac{-s_{1}}{-s_{2}}\right)}{\left(s_{1}-s_{2}\right)^{2}} \rightarrow \frac{\mathrm{L}_{1}\left(\frac{-s_{1}}{-s_{2}}\right)}{s_{2}^{2}},
$$

where $\mathrm{L}_{1}(r) \equiv\left(\mathrm{L}_{0}(r)+1\right) /(1-r), \mathrm{L}_{0}(r) \equiv \ln (r) /(1-r)$. Subtracting from the full amplitude all the cut-containing terms, and those rational functions linked to them by spurious singularities, defines a 'pure-rational' remainder. This function may be computed by on-shell recurrent techniques as done at tree level by BCFW [37], and at one loop in the present paper. To analyze the factorization properties, that is the residues at the poles in $z$, it may prove useful to think of the pure-rational remainder as the difference between the scalar loop amplitude in $D=4-2 \epsilon$ and that in $D=4$. Terms in the loop integral which are proportional to the $(-2 \epsilon)$ components of the loop momentum are well-behaved in the infrared. For this reason, complications (discontinuities) in amplitude factorization which are associated with infrared divergences [46] should be absent from the appropriate differences of amplitudes.

One may wonder whether one can push this approach further. Indeed, it seems quite likely that it will apply at tree level to a wide variety of field theories, including scalar field theories or gauge theories with massive fermions or scalars, and indeed even to spontaneously-broken gauge theories. Another question is to what extent it can be applied beyond tree level, and indeed beyond the rational terms at loop level as discussed above. At loop level, the amplitude will now have branch cuts in $z$; but in general, we may still expect it to fall off at 
infinity, allowing $A(0)$ to be analyzed in terms of simpler (lower-point) amplitudes. We are optimistic that these techniques will open the door to many new computations of Standard Model scattering amplitudes.

\section{Acknowledgments}

We thank Ed Witten, Peter Svrček and Valya Khoze for helpful conversations and comments, and Freddy Cachazo for a relevant comment. We also thank the Mathematical Institute at Oxford University, where this work was begun, and Academic Technology Services at UCLA for computer support.

[1] E. W. N. Glover, Nucl. Phys. Proc. Suppl. 116:3 (2003) [hep-ph/0211412];

C. Anastasiou, L. Dixon, K. Melnikov and F. Petriello, Phys. Rev. D 69:094008 (2004) [hep$\mathrm{ph} / 0312266]$.

[2] E. Witten, Commun. Math. Phys. 252:189 (2004) [hep-th/0312171].

[3] V. P. Nair, Phys. Lett. B214:215 (1988).

[4] R. Roiban, M. Spradlin and A. Volovich, JHEP 0404:012 (2004) [hep-th/0402016];

R. Roiban and A. Volovich, Phys. Rev. Lett. 93:131602 (2004) [hep-th/0402121];

R. Roiban, M. Spradlin and A. Volovich, Phys. Rev. D70:026009 (2004) [hep-th/0403190];

E. Witten, hep-th/0403199.

[5] N. Berkovits and E. Witten, JHEP 0408:009 (2004) [hep-th/0406051].

[6] F. Cachazo, P. Svrček and E. Witten, JHEP 0409:006 (2004) [hep-th/0403047].

[7] S. J. Parke and T. R. Taylor, Phys. Rev. Lett. 56:2459 (1986).

[8] M. L. Mangano, S. J. Parke and Z. Xu, Nucl. Phys. B298:653 (1988).

[9] F. A. Berends and W. T. Giele, Nucl. Phys. B306:759 (1988).

[10] S. Gukov, L. Motl and A. Neitzke, hep-th/0404085.

[11] I. Bena, Z. Bern and D. A. Kosower, hep-th/0406133.

[12] G. Georgiou and V. V. Khoze, JHEP 0405:070 (2004) [hep-th/0404072];

J. B. Wu and C. J. Zhu, JHEP 0409:063 (2004) [hep-th/0406146];

G. Georgiou, E. W. N. Glover and V. V. Khoze, JHEP 0407:048 (2004) [hep-th/0407027]. 
[13] D. A. Kosower, hep-th/0406175.

[14] C. J. Zhu, JHEP 0404:032 (2004) [hep-th/0403115];

J. B. Wu and C. J. Zhu, JHEP 0407:032 (2004) [hep-th/0406085];

Y. Abe, V. P. Nair and M. I. Park, Phys. Rev. D 71:025002 (2005) [hep-th/0408191].

[15] L. J. Dixon, E. W. N. Glover and V. V. Khoze, JHEP 0412:015 (2004) [hep-th/0411092];

Z. Bern, D. Forde, D. A. Kosower and P. Mastrolia, hep-ph/0412167;

S. D. Badger, E. W. N. Glover and V. V. Khoze, hep-th/0412275.

[16] F. Cachazo, P. Svrček and E. Witten, JHEP 0410:074 (2004) [hep-th/0406177].

[17] A. Brandhuber, B. Spence and G. Travaglini, Nucl. Phys. B 706:150 (2005) [hep-th/0407214].

[18] F. Cachazo, P. Svrček and E. Witten, JHEP 0410:077 (2004) [hep-th/0409245].

[19] I. Bena, Z. Bern, D. A. Kosower and R. Roiban, hep-th/0410054.

[20] F. Cachazo, hep-th/0410077.

[21] R. Britto, F. Cachazo and B. Feng, hep-th/0410179.

[22] Z. Bern, V. Del Duca, L. J. Dixon and D. A. Kosower, hep-th/0410224.

[23] R. Britto, F. Cachazo and B. Feng, hep-th/0412103.

[24] Z. Bern, L. J. Dixon and D. A. Kosower, hep-th/0412210.

[25] R. Britto, F. Cachazo and B. Feng, hep-th/0411107.

[26] Z. Bern, L. J. Dixon, D. C. Dunbar and D. A. Kosower, Nucl. Phys. B425:217 (1994) [hep$\mathrm{ph} / 9403226]$.

[27] Z. Bern, L. J. Dixon, D. C. Dunbar and D. A. Kosower, Nucl. Phys. B435:59 (1995) [hep$\mathrm{ph} / 9409265]$.

[28] Z. Bern and A. G. Morgan, Nucl. Phys. B467:479 (1996) [hep-ph/9511336];

Z. Bern, L. J. Dixon and D. A. Kosower, Ann. Rev. Nucl. Part. Sci. 46:109 (1996) [hepph/9602280];

Z. Bern, L. J. Dixon and D. A. Kosower, Nucl. Phys. Proc. Suppl. 51C:243 (1996) [hep$\mathrm{ph} / 9606378] ;$

Z. Bern, L. J. Dixon and D. A. Kosower, JHEP 0001:027 (2000) [hep-ph/0001001].

[29] Z. Bern, L. J. Dixon and D. A. Kosower, JHEP 0408:012 (2004) [hep-ph/0404293].

[30] Z. Bern, L. J. Dixon and D. A. Kosower, Nucl. Phys. B513:3 (1998) [hep-ph/9708239].

[31] R. J. Eden, P. V. Landshoff, D. I. Olive, J. C. Polkinghorne, The Analytic S Matrix, (Cambridge University Press, 1966). 
[32] F. A. Berends, W. T. Giele and H. Kuijf, Nucl. Phys. B333:120 (1990).

[33] W. T. Giele and E. W. N. Glover, Phys. Rev. D46:1980 (1992);

Z. Kunszt, A. Signer and Z. Trócsányi, Nucl. Phys. B420:550 (1994) [hep-ph/9401294];

S. Catani, Phys. Lett. B427:161 (1998) [hep-ph/9802439].

[34] R. Roiban, M. Spradlin and A. Volovich, hep-th/0412265.

[35] R. Britto, F. Cachazo and B. Feng, hep-th/0412308.

[36] M. Luo and C. Wen, hep-th/0501121.

[37] R. Britto, F. Cachazo, B. Feng and E. Witten, hep-th/0501052.

[38] Z. Bern, L. J. Dixon and D. A. Kosower, hep-th/9311026.

[39] Z. Bern, G. Chalmers, L. J. Dixon and D. A. Kosower, Phys. Rev. Lett. 72:2134 (1994) [hep$\mathrm{ph} / 9312333]$;

Z. Bern, L. J. Dixon, D. C. Dunbar and D. A. Kosower, hep-ph/9405248.

[40] J. E. Paton and H. M. Chan, Nucl. Phys. B10:516 (1969);

P. Cvitanović, P. G. Lauwers and P. N. Scharbach, Nucl. Phys. B186:165 (1981);

F. A. Berends and W. Giele, Nucl. Phys. B294:700 (1987);

D. Kosower, B. H. Lee and V. P. Nair, Phys. Lett. B201:85 (1988).

[41] M. L. Mangano and S. J. Parke, Phys. Rept. 200:301 (1991);

L. J. Dixon, in QCD $\&$ Beyond: Proceedings of TASI '95, ed. D. E. Soper (World Scientific, 1996) [hep-ph/9601359].

[42] F. A. Berends, R. Kleiss, P. De Causmaecker, R. Gastmans, and T. T. Wu, Phys. Lett. 103B:124 (1981);

P. De Causmaeker, R. Gastmans, W. Troost, and T. T. Wu, Nucl. Phys. B206:53 (1982);

Z. Xu, D.-H. Zhang, L. Chang, Tsinghua University preprint TUTP-84/3 (1984), unpublished;

R. Kleiss and W. J. Stirling, Nucl. Phys. B262:235 (1985);

J. F. Gunion and Z. Kunszt, Phys. Lett. 161B:333 (1985);

Z. Xu, D.-H. Zhang, and L. Chang, Nucl. Phys. B291:392 (1987).

[43] Z. Bern and D. A. Kosower, Nucl. Phys. B362:389 (1991).

[44] G. Mahlon, Phys. Rev. D49:4438 (1994) [hep-ph/9312276].

[45] Z. Bern, L. J. Dixon and D. A. Kosower, Phys. Rev. Lett. 70:2677 (1993) [hep-ph/9302280].

[46] Z. Bern and G. Chalmers, Nucl. Phys. B447:465 (1995) [hep-ph/9503236].

[47] Z. Bern, V. Del Duca and C. R. Schmidt, Phys. Lett. B 445:168 (1998) [hep-ph/9810409]; 
D. A. Kosower and P. Uwer, Nucl. Phys. B563:477 (1999) [hep-ph/9903515];

Z. Bern, V. Del Duca, W. B. Kilgore and C. R. Schmidt, Phys. Rev. D60:116001 (1999) [hep-ph/9903516].

[48] Z. Bern and D. A. Kosower, Nucl. Phys. B379:451 (1992).

[49] Z. Bern, L. J. Dixon and D. A. Kosower, Nucl. Phys. B437:259 (1995) [hep-ph/9409393].

[50] C. Quigley and M. Rozali, hep-th/0410278;

J. Bedford, A. Brandhuber, B. Spence and G. Travaglini, Nucl. Phys. B706:100 (2005) [hepth/0410280].

[51] S. J. Bidder, N. E. J. Bjerrum-Bohr, L. J. Dixon and D. C. Dunbar, Phys. Lett. B606:189 (2005) [hep-th/0410296];

S. J. Bidder, N. E. J. Bjerrum-Bohr, D. C. Dunbar and W. B. Perkins, hep-th/0412023.

[52] J. Bedford, A. Brandhuber, B. Spence and G. Travaglini, hep-th/0412108.

[53] Note that a version of the 'odd' terms $O_{n}$ in the first reference in ref. [39] (the last line of eq. (7)) has the wrong sign. 